\title{
Amiloidose localizada laríngea: relato de caso e revisão de literatura
}

\section{Localized laryngeal amyloidosis: case report and literature review}

\author{
Fabiana C. P. Valera', Denílson S. Fomin ${ }^{1}$, \\ Gilberto S. Maggioni Jr. ${ }^{2}$, Marcos Grellet
}

Palavras-chave: amiloidose, laringe.

Key words: amyloidosis, larynx.

\section{Resumo / Summary}

\begin{abstract}
A
amiloidose localizada laríngea é uma doença rara, correspondendo a menos de 1\% dos tumores benignos da laringe. No entanto, a amiloidose localizada acomete principalmente a laringe nas vias aéreas, sendo assim de grande importância para o otorrinolaringologista. Ela está relacionada à produção monoclonal de imunoglobulinas de cadeia leve, principalmente $1 \mathrm{e} \mathrm{k}$. Este estudo tem como objetivo relatar um caso de amiloidose laríngea atendida no Ambulatório de Laringologia e Voz do Hospital das Clínicas da FMRP - USP, assim como discutir a revisão de literatura.
\end{abstract}

\begin{abstract}
Cocalized laryngeal amyloidosis is a rare disease, corresponding to less than $1 \%$ of benign tumors in larynx. However, localized amyloidosis occurs mostly in larynx, being of great importance its diagnose by Otorhinolaryngologists. It is related to monoclonal production of light chain immunoglobulins, mainly $\mathrm{l}$ and $\mathrm{k}$. This study has the purpose of relating a case of laryngeal amyloidosis assisted in Laryngology and Voice Clinics at Clinics Hospital FMRP - USP, and discussing literature as well.
\end{abstract}

\footnotetext{
${ }^{1}$ Médicos associados da Disciplina de Otorrinolaringologia da Faculdade de Medicina de Ribeirão Preto - Universidade de São Paulo.

${ }^{2}$ Médico associado da Disciplina de Patologia da da Faculdade de Medicina de Ribeirão Preto - Universidade de São Paulo. ${ }^{3}$ Professor livre-docente e Associado da Faculdade de Medicina de Ribeirão Preto - Universidade de São Paulo. Instituição: Hospital das Clínicas da Faculdade de Medicina de Ribeirão Preto- Universidade de São Paulo Endereço para Correspondência: Av. Bandeirantes, 3900 Bairro Monte Alergre Ribeirão Preto SP E-mail: facpvalera@uol.com.br

Artigo recebido em 26 de maio de 2003. Artigo aceito em 08 de agosto de 2003.
} 


\section{INTRODUÇÃO E REVISÃO DE LITERATURA}

Amiloidose se caracteriza por um grupo de doenças de depósito de proteínas que possuem natureza fibrilar e propriedades tintoriais específicas. Pode ser classificada em localizada ou sistêmica, e de acordo com os precursores protéicos fibrilares mais importantes. A classificação mais aceita atualmente é a da Sociedade Internacional de Amiloidose, em 1990'르 que divide a amiloidose em:

- tipo AA (ou secundária): composta pela amiloidose sistêmica reativa, associada a doenças inflamatórias crônicas e ocasionalmente a neoplasias, e pela febre de Mediterrâneo familiar. Formada pelo amilóide sérico A.

- tipo AL (ou primária): formada por cadeias monoclonais de imunoglobulina de cadeia leve $\kappa$ ou $\lambda$. Composta pela amiloidose sistêmica primária, pela amiloidose associada a mieloma, gamopatia monoclonal ou discrasia oculta e pela amiloidose localizada.

- tipo ATTR: composta pela amiloidose sistêmica senil, e pelas polineuropatia e cardiomiopatia amiloidais familiares. Formada pela transtiretina (ou pré-albumina).

- tipo $\mathrm{A} \beta_{2} \mathrm{M}$ : formada pela microglobulina $\beta_{2}$ Composta pela amiloidose periarticular e pela amiloidose sistêmica associada a insuficiência renal e diálise por período prolongado.

- tipo $A \beta$ : formada pela precursora protéica $\beta$. Composta pela doença de Alkheimer e alguns casos de Síndrome de Down.

A amiloidose localizada é uma doença rara (correspondendo a menos de $1 \%$ dos tumores benignos de larin$\mathrm{ge}^{2}$ ) e de causa desconhecida. Localiza-se principalmente em vias aerodigestivas ${ }^{3,4}$ (sobretudo em laringe) e pele ${ }^{5}$.

Desde 1875, quando Burow e Neumann primeiro descreveram a amiloidose laríngea, pouco mais de 300 casos foram relatados em literatura ${ }^{6}$. Apesar da amiloidose laríngea ser rara, a laringe é o local mais comumente acometido pela amiloidose localizada, sobretudo em região supraglótica ${ }^{7}$.

\section{APRESENTAÇÃO DE CASO CLÍNICO}

UMPC, 33 anos, sexo feminino, procurou o Hospital das Clínicas da Faculdade de Medicina de Ribeirão Preto com história de disfonia há 6 meses, sem dispnéia, apnéias, distúrbios de deglutição ou outros sintomas. Negava história de abuso vocal, tabagismo ou perda rápida de peso neste período.

Ao exame fibroscópico, evidenciava-se lesão subepitelial amarelada nodular pequena em comissura anterior, sem outras alterações em laringe (Figura 1).

Em 1999, a paciente foi submetida à biópsia sob laringoscopia direta, e a biópsia demonstrou um extenso e denso material amorfo eosinofílico, corado para vermelho Congo, o que confere o diagnóstico de amiloidose laríngea (Figuras $2 \mathrm{a}$ e $2 \mathrm{~b}$ ).

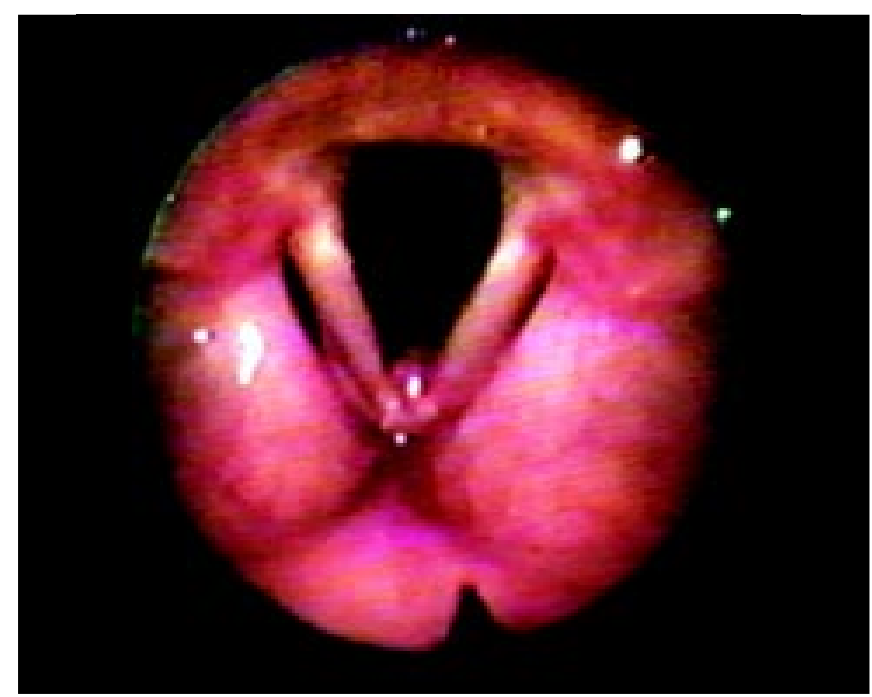

Figura 1. Imagem endoscópica da laringe da paciente, evidenciando lesão amilóide em comissura anterior de pregas vocais.
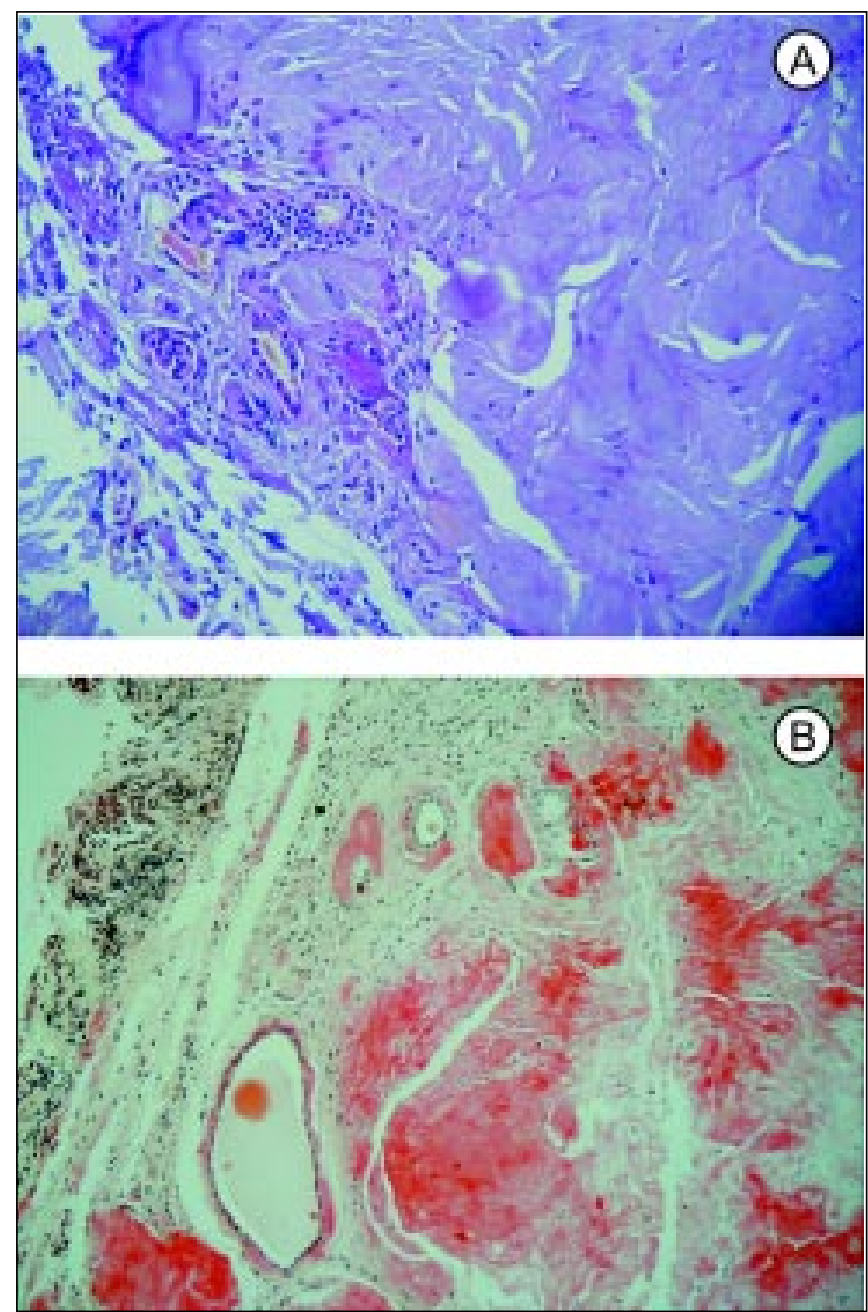

Figuras 2a e 2b. Biópsias de tecido amilóide laríngeo: colorações Hematoxilina-Eosina (Figura 2a) e Vermelho Congo (Figura 2b). 
Foi realizada tomografia computadorizada de pescoço, que evidenciou discreto espessamento a nível de comissura anterior, sem acometimento das demais porções da laringe e terço superior da traquéia, e ausência de adenomegalias.

Foram realizados exames para se descartar amiloidose sistêmica (radiografia de tórax e abdome, ultrassom abdominal, funções hepática e renal, glicemia, proteína $\mathrm{C}$ reativa, eletroforese de proteínas, urina rotina e biópsia de gordura umbilical), doenças imunológicas (fator reumatóide, ANCA e ANA) e mieloma múltiplo (proteína de Bence Jones), tendo sido todos considerados dentro da normalidade.

Devido pequeno tamanho residual da lesão após biópsia e ao comportamento benigno da doença na maioria dos casos, optou-se por seguimento clínico mensal da paciente por seis meses a após semestral, com fibroscopias a cada retorno. A paciente permanece atualmente com apenas leve disfonia, mantendo-se sem outros sintomas. A última fibroscopia, três anos e meio após diagnóstico inicial, demonstrou discreta sinéquia em comissura anterior, sem aumento de dimensões da lesão.

\section{DISCUSSÃO}

A amiloidose localizada laríngea, do tipo AL, tem como precursora a discrasia monoclonal de células plasmáticas, o que leva à formação de subunidades protéicas de fibrilas derivadas de imunoglobulina de cadeia leve $(\lambda \text { ou } \kappa)^{5}$, sendo a cadeia leve $\lambda$ mais propensa e formar amilóides do que a cadeia $\kappa$.

A amiloidose pode ocorrer pela produção de imunoglobulinas tanto estruturalmente anormais ou em quantidade excessiva pelas células plasmáticas monoclonais. Os depósitos amilóides ocorreriam pela dificuldade em metabolizar as fibrilas produzidas.

A laringe é o local mais comumente acometido pela amiloidose localizada, em especial em pregas vestibulares. A localização múltipla em vias aéreas pode acontecer, sendo mais freqüente o acometimento associado em traquéia ${ }^{8}$.

O sintoma mais comum é da amiloidose laríngea é a disfonia, raramente sendo observados outros sintomas, como dispnéia, odinofagia, estridor inspiratório aspirações, engasgos ou plenitude faríngea.

O exame da lesão deve compreender os exames endoscópicos, a laringoscopia direta e os exames de imagem (ressonância magnética ou, preferencialmente, a tomografia computadorizada) para se identificar a extensão da lesão. À fibroscopia, o aspecto é de lesão nodular amarelada, não ulcerada, com depósitos subepiteliais.

O diagnóstico é confirmado com biópsia, evidenciando-se depósitos amilóides extracelulares homogêneos, amorfos e eosinofílicos. Durante a coloração de Vermelho Congo, observa-se a típica birrefrigência esverdeada à microscopia de polarização.

A imunohistoquímica evidencia depósitos amilóides monoclonais para imunoglobulinas de cadeias leves $\kappa$ ou $\lambda$, enquanto as células plasmáticas produtoras das imunoglobulinas possuem padrão policlonal para cadeias tanto $\kappa$ como $\lambda^{8}$.

Devido à evolução lenta e aos sintomas discretos da amiloidose localizada, o tratamento de escolha é a retirada da lesão via endoscópica (preferencialmente com uso de laser de dióxido de carbono), apenas para os casos sintomáticos ${ }^{5,6,7,8}$. O objetivo inicial da cirurgia deve ser somente a retirada do amilóide visível, sem ressecções amplas.

Em raros casos, onde a extensão da doença é muito ampla, ou em casos de recidivas freqüentes, indica-se a ressecção cirúrgica externa. Kennedy et al. ${ }^{6}$ sugerem que a cirurgia externa mais adequada seja a cirurgia externa supraglótica lateral, por ser ela mais conservadora, evitando assim complicações como alterações na deglutição e na voz.

A maioria dos autores em literatura ${ }^{6,8-11}$ concorda que o tratamento com imunomoduladores (quimioterápicos, corticosteróides e radioterapia) deve ser evitado porque o comprometimento da resposta imune do paciente pode acelerar o processo de depósito amilóide.

Segundo Lewis et al. ${ }^{8}$, a recorrência da amiloidose localizada é variável, podendo ocorrer até vários anos após a primeira cirurgia. Ela é mais expressiva nos casos em que se observa ao diagnóstico maior extensão da lesão. No entanto, são raros os casos que evoluem a óbito devido obstrução respiratória ou que apresentam amiloidose sistêmica ${ }^{2,6,8}$.

\section{COMENTÁRIOS FINAIS}

A amiloidose laríngea é uma entidade rara, contudo deve ser pensada como diagnóstico de lesões benignas em laringe. A conduta deve ser sempre a menos agressiva o possível, indicando-se cirurgia para apenas os casos sintomáticos. O seguimento clínico deve ser realizado por longos períodos, pois as recidivas podem ocorrer tardiamente.

\section{REFERÊNCIAS BIBLIOGRÁFICAS}

1. Husby G, Araki S, Benditt EP et al. The 1990 guidelines for nomenclature and classification of amyloid and amyloidosis. In: Natvig JB, Förre Husby $G$ et al, eds. Amyloid and amyloidosis. Vith International Symposium on Amyloidosis. Norway. Dordrecht: Kluwer Acamedic; 1990: 5-11.

2. Godbersen GS, Leh JF, Hansmann ML, Rudert H, Linke RP. Organlimited laryngeal amyloid deposits: clinical, morphological and immunohistochemical results of five cases. Ann Otol Rhinol Laryngol 1992; 101: 770-5.

3. Simpson GT II, Strong MS, Skinner M, Cohen AS. Localized amyloidosis of the head and neck and upper aerodigestive and lower respiratory tracts. Ann Otol Rhinol Laryngol 1984; 93: 3749 .

4. Mufarrij AA, Busaba NY, Zaytoun GM, Gallo GR, Feiner HD. Primary localized amyloidosis of the nose and paranasal sinuses. Am J Surg Pathol 1990; 14: 379-83. 
5. Berg AM, Troxler RT, Grillone G, Kanznica J, Kane K et al. Localized amyloidosis of the larynx: evidence for light chain composition. Ann Otol Rhinol Laryngol 1993; 102: 884-9.

6. Kennedy TL, Patel NM. Surgical management of localized amyloidosis. Laryngoscope 2000; 110: 918-23.

7. Clark JM, Weissler MC. Localized laryngotracheobronchial amyloidosis: case report nd review of the literature. Ear Nose Throat J 2001; 80: 632-7.

8. Lewis JE, Olsen KD, Kurtin PJ, Kyle RA. Laryngeal amyloidosis: a clinicopathologic and immunohistochemical review. Otolaryngol. Head Neck Surg 1992; 106: 372-7.
9. Mitrani M. Biller HF. Laryngeal amyloidosis. Laryngoscope 1985; 95: $1346-7$

10. Rubinow A, Celli BR, Cohen AS et al. Localized mayloidosis of the lower respiratory tract. Am Rev Respir Dis 1978; 118: 60311.

11. Friedman I. Nose, throat and ears. In: Symmers WS, ed. Systemic Pathology. 3rd ed. New York; Edinburgh: Churchill Livingstone; 1987: $203-5$. 